\title{
EFEITO DA ADIÇÃO DE Nb NA MICROESTRUTURA DE FERRO FUNDIDO BRANCO ALTO CROMO HIPEREUTÉTICO*
}

\author{
Dimas de Andrade Pacheco ${ }^{1}$ \\ Geralda Cristina Durães de Godoy² \\ Cláudio Gonçalves de Oliveira ${ }^{3}$ \\ Loudiana Mosqueira Antônio ${ }^{4}$ \\ Mário José Bueno
}

\section{Resumo}

O estudo do efeito da adição de nióbio em um ferro fundido branco alto cromo tratado termicamente com $25 \% \mathrm{Cr}$ e $3 \% \mathrm{C}$ foi estudado neste trabalho por microscopia ótica, microscopia eletrônica de varredura e difração de raios $X$. Partindo de uma liga hipereutética, a adição de $2 \%$ de nióbio foi definida considerando que todo $\mathrm{Nb}$ adicionado se encontraria na forma de carbonetos de nióbio, $\mathrm{NbC}$, e com o objetivo de obter uma microestrutura próxima à eutética. $\mathrm{A}$ adição promoveu um refinamento da microestrutura, sendo que uma fração volumétrica de $27 \%$ de carbonetos $\mathrm{M}_{7} \mathrm{C}_{3}$ foi observada. Carbonetos grosseiros, inicialmente formados, não são facilmente observados. Uma microestrutura formada por $\mathrm{NbC}$ e eutético, $\mathrm{M}_{7} \mathrm{C}_{3}+$ matriz (austenita ou martensita), foi obtida. Os carbonetos de nióbio formados são encontrados principalmente na forma compacta. É esperado, portanto, que, a liga com adição de $2 \%$ de nióbio apresente melhor resistência ao desgaste abrasivo que a liga sem nióbio, em razão dos carbonetos primários, frágeis e grosseiros, terem sido praticamente suprimidos após a adição de $\mathrm{Nb}$ e uma microestrutura mais refinada tenha sido obtida.

Palavras-chave: Ferro fundido branco alto cromo; hipereutético; nióbio; tribologia; desgaste abrasivo; mineração.

\section{EFFECT OF Nb ADDITION ON THE MICROSTRUCTURE OF HYPEREUTETIC HIGH CHROMIUM WHITE CAST IRON}

\begin{abstract}
The effect of niobium addition on heat treated high chromium white cast containing $25 \mathrm{wt} \% \mathrm{Cr}$ and $3 w t \% \mathrm{C}$ was studied in this work by optical microscopy, scanning electron microscopy and X-ray diffraction. Starting from a hypereutectic alloy, the addition of $2 \%$ niobium was stablished considering that all $\mathrm{Nb}$ would be found as $\mathrm{NbC}$, niobium carbides, and aiming a microstructure close to the eutectic one. The addition refined the microstructure, with a carbide volume fraction of $27 \%$ being observed. Coarse carbides, initially formed, are hardly observed. A microstructure formed by $\mathrm{NbC}$ and eutectic microstructure, $\mathrm{M}_{7} \mathrm{C}_{3}+$ matrix (austenitic or martensitic), was obtained. Niobium carbides are mostly found in the compact form. It is expected, therefore, that the alloy with $\mathrm{Nb}$ addition exhibits better abrasive wear resistance than the alloy without addition, on account of the primary carbides, brittle and coarse, have been suppressed after niobium addition and a more refined microstructure has been obtained.
\end{abstract}

Keywords: High chromium white cast iron; hypereutetic; niobium; tribology; abrasive wear; mining.

Engenharia Metalúrgica, Mestrando, DEMET, UFMG, Belo Horizonte - MG, Brasil.

Engenharia Metalúrgica e de Minas, Doutora, Professora Titular, DEMET, UFMG, Belo Horizonte - MG, Brasil.

Engenharia de Materiais, Mestre, Douturando, DEMET, UFMG, Belo Horizonte - MG, Brasil.

Engenharia Metalúrgica, Mestranda, DEMAT, CEFET MG, Belo Horizonte - MG, Brasil.

Ciência e Tecnologia de Radiações, Minerais e Materiais, Mestre, Doutorando, DEMET, UFMG, Belo Horizonte - MG, Brasil. 


\section{INTRODUÇÃO}

Os ferros fundidos branco alto cromo (FFACr) são amplamente utilizados em aplicações onde a resistência ao desgaste abrasivo é essencial e a capacidade necessária para suportar impacto é moderada [1]. Na mineração são aplicados nas mais diversas condições de desgaste, como britagem, moagem, calhas, chutes e bombeamento de polpa. Os FFACr podem assumir uma microestrutura hipoeutética, eutética ou hipereutética. Sua microestrutura hipereutética é formado por carbonetos primários $\mathrm{M}_{7} \mathrm{C}_{3}$ e regiões de eutético (austenita e $\mathrm{M}_{7} \mathrm{C}_{3}$ ) [1,2]. A resistência à abrasão dessas ligas é principalmente atribuída à presença destes carbonetos de alta dureza, entre 1200 e 1800 HV. Entretanto, os carbonetos primários, presentes na microestrutura hipertética, são grosseiros e frágeis e podem influenciar negativamente o desempenho ao desgaste abrasivo, principalmente em situações de alto impacto [1-3], onde o mecanismo de fratura frágil pode diminuir a resistência ao desgaste abrasivo desses materiais. Geralmente, uma melhor resistência ao desgaste abrasivo é obtida nos FFACr quando uma microestrutura eutética é observada, com uma fração volumétrica de carbonetos $\mathrm{M}_{7} \mathrm{C}_{3}$ de aproximadamente $30 \%$ [3-7].

A adição de nióbio, assim como outros elementos formadores de carbonetos, é um recurso que pode ser utilizado para melhoria das propriedades dos FFACr. O nióbio promove o refinamento da microestrutura hipereutética, pela depleção de carbono no líquido devido a formação de carbonetos $\mathrm{NbC}$, mais finos e de maior dureza que os $\mathrm{M}_{7} \mathrm{C}_{3}$. Alterações na morfologia, fração volumétrica e tamanho dos carbonetos primários também são observadas. Os FFACr apresentam melhor resistência ao desgaste, em diferentes condições e testes, após a adição de Nb [3,7-9]. Melhorias na resistência à abrasão também são obtidas por tratamento térmico, produzindo uma transformação da austenita em martensita $[1,10]$.

O objetivo desse trabalho é, por meio da adição de nióbio, obter uma microestrutura eutética refinada em um ferro fundido branco alto cromo hipereutético, reduzindo assim a quantidade de carbonetos $\mathrm{M}_{7} \mathrm{C}_{3}$ primários frágeis e deletérios à resistência ao desgaste abrasivo.

\section{MATERIAIS E MÉTODOS}

As ligas estudadas foram produzidas em forno de indução e fundidas em barras de $75 \times 25 \times 12,7 \mathrm{~mm}$. A composição da liga inicial, sem adição de nióbio, foi baseada em ligas hipereutéticas, ASTM A532 classe III tipo A, utilizadas na mineração como placas de desgaste e apresentam bom rendimento operacional. O teor de nióbio a ser adicionado foi definido afim de se buscar uma microestrutura eutética [3], considerando que o nióbio adicionado formará carbonetos $\mathrm{NbC}$ e sua presença nas outras fases será insignificante. A composição química das ligas, sem e com adição de $\mathrm{Nb}$, está apresentado na Tabela 1.

Tabela 1 - Composição química em \% massa das ligas estudadas

\begin{tabular}{lcccccccc}
\hline & $\mathbf{C}$ & $\mathbf{C r}$ & $\mathbf{M n}$ & $\mathbf{C u}$ & $\mathbf{N i}$ & $\mathbf{S i}$ & Mo & Nb \\
\hline FFACr & 3,1 & 25,0 & 0,9 & 0,1 & 0,8 & 1,0 & 0,1 & - \\
\hline FFACrNb & 3,1 & 24,6 & 0,8 & 0,1 & 1,1 & 1,1 & 0,1 & 2,0 \\
\hline
\end{tabular}

Um tratamento térmico que consiste em recozimento a $730^{\circ} \mathrm{C}$ por 2,5 horas; seguido de desestabilização da austenita a $1020^{\circ} \mathrm{C}$ por 2,5 horas (diminuindo o carbono em 
solução pela formação de carbonetos secundários e resultando no aumento da temperatura $\mathrm{M}_{\mathrm{s}}$ e $\mathrm{M}_{\mathrm{f}}$ ) [1]; resfriamento forçado a ar; e revenimento a $250^{\circ} \mathrm{C}$ por 2 horas foi realizado nas duas ligas. A rampa de aquecimento e resfriamento utilizada, exceto para o resfriamento forçado a ar, foi de $110^{\circ} \mathrm{C} / \mathrm{h}$.

A caracterização estrutural das ligas foi realizada por difratometria de Raios $X$ (DRX), microscopia ótica (MO) e microscopia eletrônica de varredura (MEV). As amostras preparadas para as técnicas acima foram retiradas de cortes transversais próximo ao centro das barras fundidas. A preparação seguiu as seguintes etapas: embutimento, lixamento, polimento e ataque químico, quando necessário. $O$ reagente Vilela (1g ácido pícrico, $5 \mathrm{ml} \mathrm{HCl}$ concentrado, $100 \mathrm{ml}$ álcool etílico PA) foi utilizado para o ataque químico. Para cálculo da fração volumétrica dos carbonetos, $\mathrm{M}_{7} \mathrm{C}_{3}$ Nital $10 \%$ foi utilizado como reagente.

A caracterização da estrutura cristalina das ligas foi realizada utilizando difratometro PANALYTICAL, modelo Empyrean, detector de estado sólido X'Celerator e radiação de CuKa $(\lambda K \alpha=1,54184 \AA$ e $\lambda K \alpha 1=1,54056 \AA)$.

Um Microscópio LEITZ/LEICA METALLUX 2, com câmera LEICA DFC290 foi utilizado para caracterizão por microscopia ótica. Já para microscopia eletrônica de varredura, foi utilizado o equipamento FEI Inspect@ S50. Análise complementar foi realizada por Espectrômetro de Energia Dispersiva (EDS) EDAX Genesis $\AA$, acoplado ao MEV. A fração volumétrica dos carbonetos $\mathrm{M}_{7} \mathrm{C}_{3}$ e $\mathrm{NbC}$ e o tamanho médio dos carbonetos de nióbio formam calculados usando o software ImageJ [11].

\section{RESULTADOS E DISCUSSÃO}

O efeito da adição de nióbio na microestrutura de um FFAC hipereutético é mostrado na Figura 1. Na liga FFACr, Figura 1 a, são observados carbonetos primários e eutéticos $\mathrm{M}_{7} \mathrm{C}_{3}$ e uma matriz martensítica. Os carbonetos primários grosseiros, facilmente observados para FFACr, não são observados após a adição de nióbio, FFACrNb, Figura 1 b. A adição de $\mathrm{Nb}$ tornou a microestrutura mais fina. Foi observado na liga FFACrNb uma microestrutura formada por carbonetos $\mathrm{NbC}$, carbonetos $\mathrm{M}_{7} \mathrm{C}_{3}$ eutéticos e duas matrizes diferentes, uma martensítica, mais escura, e outra austenítica.

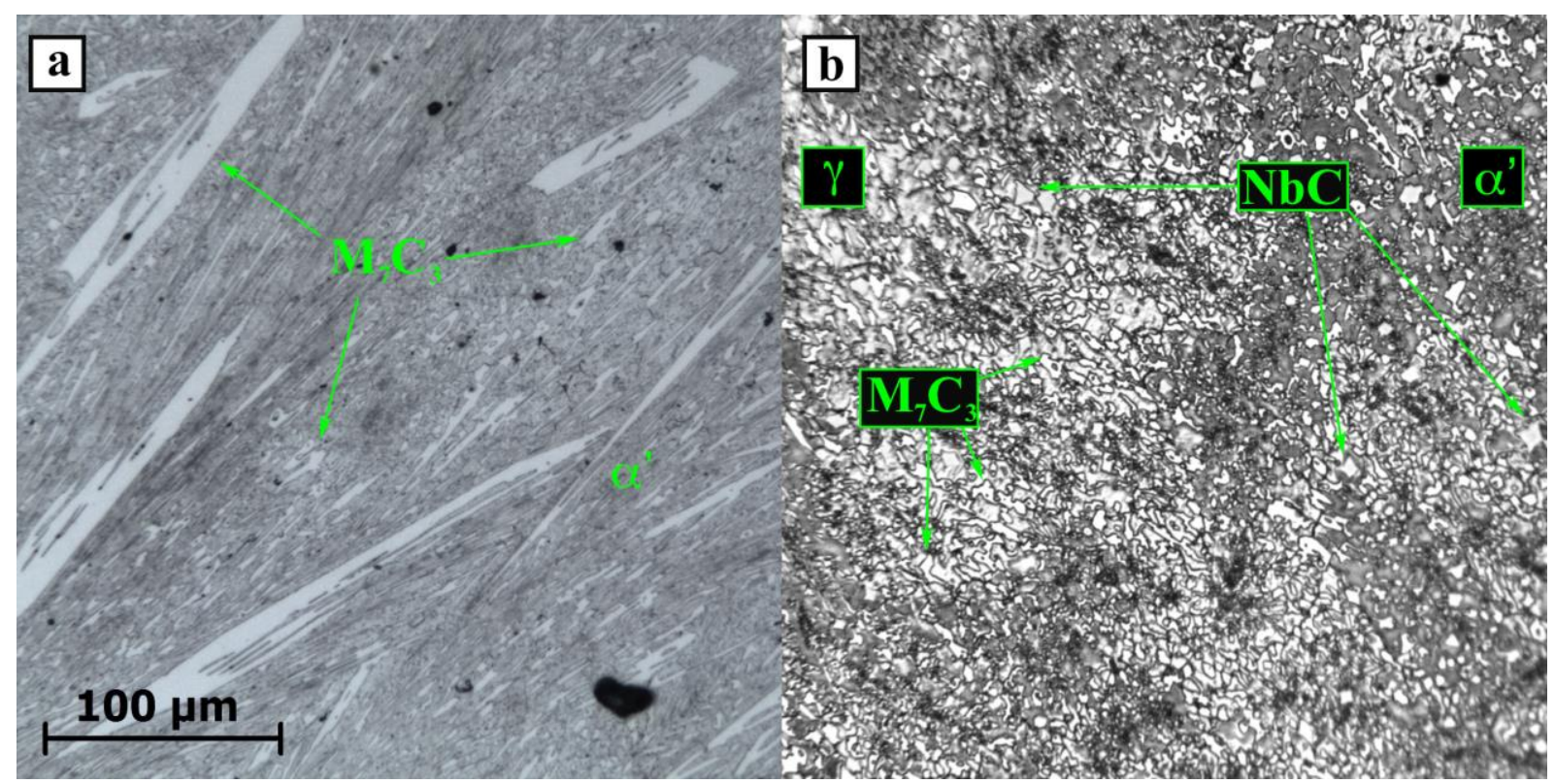

Figura 1 - Efeito da adição de nióbio na microestrutura hipereutética: (a) FFACr e (b) FFACrNb 
Os resultados obtidos por DRX, Figura 2, corroboram com a microscopia óptica. A adição de nióbio na liga 2 levou a formação de NbC. Além disso, os resultados indicam que o tratamento térmico não proporcionou uma transformação total da austenita (FecFc), principalmente para a liga 2, sendo observado Feccc (martensita), FecFc (austenia) e $\mathrm{M}_{7} \mathrm{C}_{3}$, nas duas ligas. Para liga 1, foi observado a presença de carbonetos secundários. Esses podem ser vistos na Figura 3, micrografia obtida por microscopia ótica da liga FFACr.

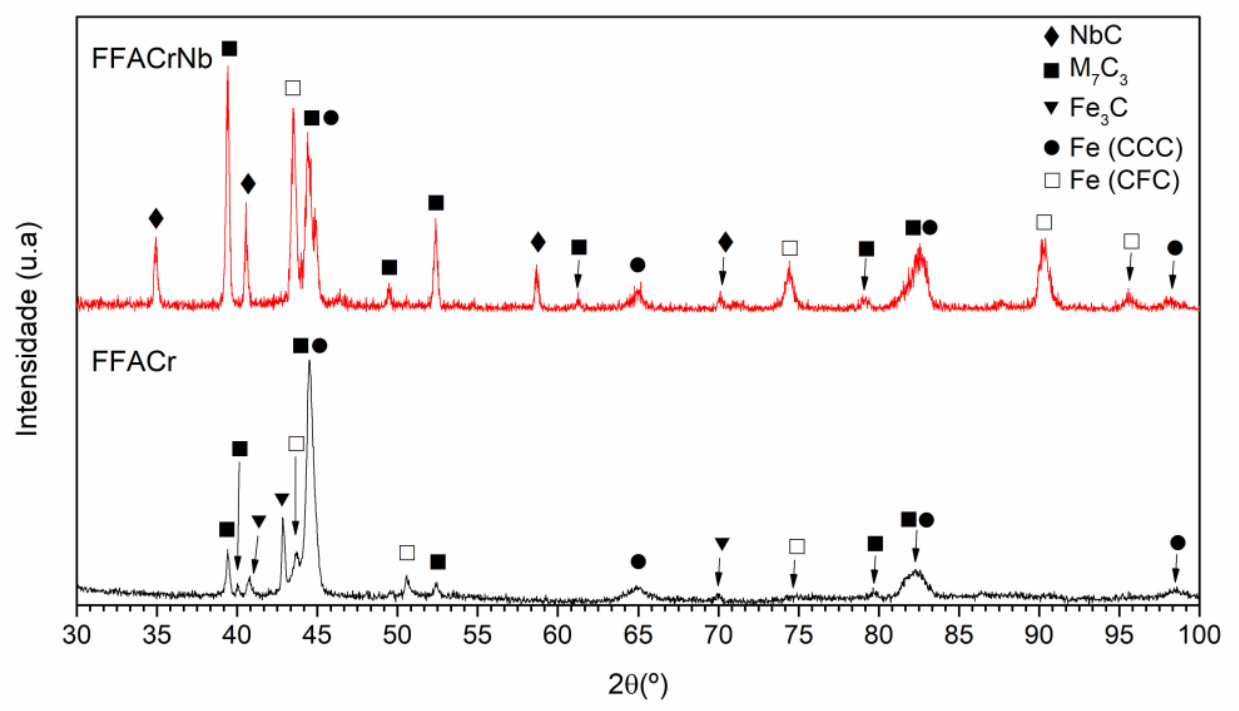

Figura 2 - Gráfico normalizado de Difração de Raios X das ligas FFACr e FFACrNb

O teor de austenita retida após a têmpera a ar forçado é muito dependente do teor de carbono na matriz após a desestabilização, que depende fortemente da temperatura da desestabilização e da seção da peça. A eficiência do tratamento térmico, buscando uma matriz martensítica, pode ser melhorada diminuindo a temperatura de tratamento. Uma menor temperatura de desestabilização levaria a um menor teor de carbono na austenita e, consequentemente, uma temperatura $\mathrm{M}_{\mathrm{s}}$ maior, o que facilitaria a transformação martensítica em têmpera a ar [1]. O efeito da temperatura de desestabilização no teor da austenita retida é menos perceptível em peças de maior seção devido a precipitação adicional de carbonetos secundários durante o lento resfriamento [12]. 


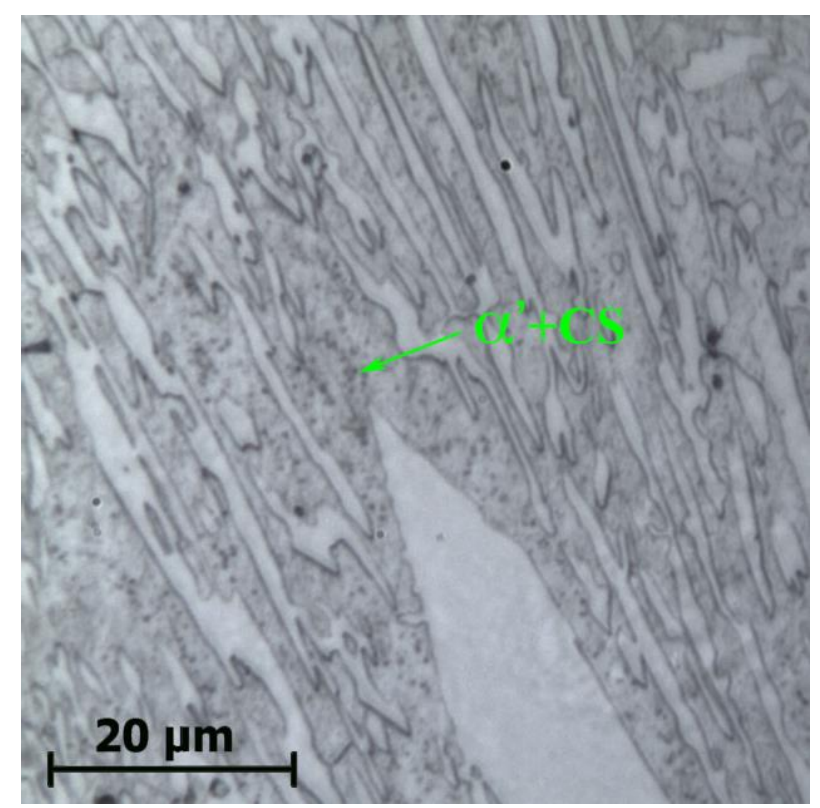

Figura 3 - Detalhe da microestrutura da liga FFACr sem adição de nióbio

A imagem obtida por elétrons retroespalhados da liga FFACrNb por MEV é mostrada na Figura 4 a. Os carbonetos de nióbio são facilmente observados. A distribuição dos $\mathrm{NbC}$ é homogênea e encontram-se principalmente em sua forma compacta. Além dos carbonetos compactos, um segundo tipo de $\mathrm{NbC}$, alongados e finos, é observado. Diferentemente dos carbonetos compactos, que são formados a temperatura superior ao da transformação eutética, esses são formados pela segregação de $\mathrm{Nb}$ no líquido durante a solidificação $[13,14]$. A presença destes pode estar ligada as dimensões do corpo de prova fundido, que promovem uma alta taxa de resfriamento da peça fundida. $\mathrm{O}$ espectro de EDS do carboneto NbC é mostrado na Figura 4 b. $O$ Ti observado pode ser proveniente de contaminação durante 0 processo de fusão da liga. Os demais elementos, presentes em menor quantidade no espectro, se devem ao fato de a resposta do método conter informações provenientes de regiões mais internas.

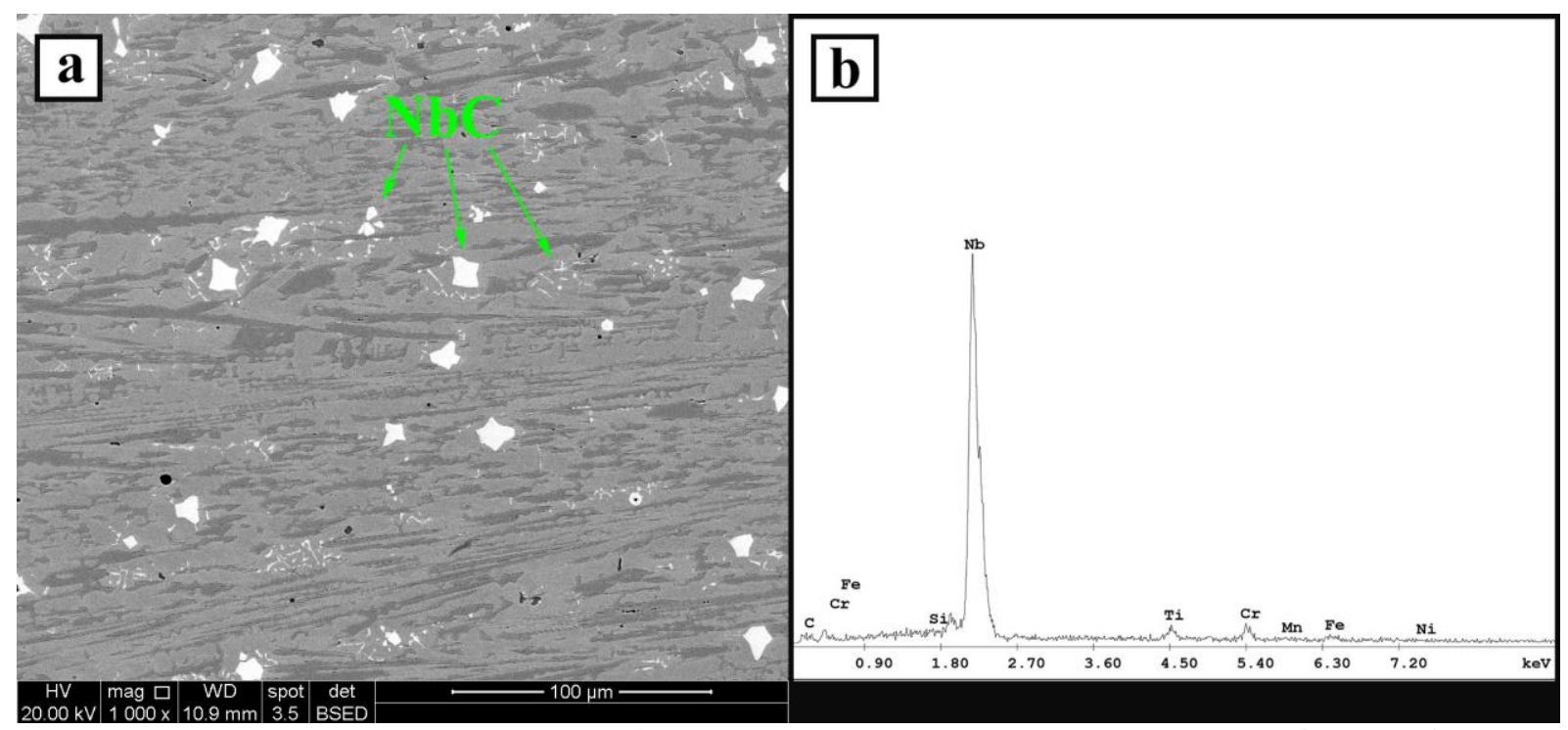

Figura 4 - Micrografia MEV por elétrons retroespalhados da Liga 2 (a) e EDS de NbC(b) 
A fração volumétrica dos carbonetos $\mathrm{M}_{7} \mathrm{C}_{3}$ e $\mathrm{NbC}$, assim como o tamanho médio dos carbonetos compactos, são apresentados na Tabela 2. A adição de nióbio levou a uma redução da fração volumétrica de carbonetos, de 41 para $27 \%$. A adição também proporcionou a precipitação de uma fração volumétrica de $2,5 \%$ de carbonetos de nióbio, sendo que $64 \%$ desses na forma compacta. O tamanho médio destes foi $0,07 \mathrm{~mm}^{2}$, de tamanho próximo aos $\mathrm{M}_{7} \mathrm{C}_{3}$ refinados, como pode ser observado na Figura 4 a. A adição de nióbio promove a redução do teor de carbono no líquido durante a solidificação, reduzindo assim a fração volumétrica e o tamanho dos carbonetos primários $\mathrm{M}_{7} \mathrm{C}_{3}$ [8].

Tabela 2 - Fração volumétrica dos carbonetos $\mathrm{M}_{7} \mathrm{C}_{3}$ e $\mathrm{NbC}$ e tamanho médio e fração dos $\mathrm{NbC}$ compactos

\begin{tabular}{lcccc}
\hline & $\begin{array}{c}\text { Fração } \\
\text { Volumétrica } \mathbf{M}_{7} \mathrm{C}_{3}\end{array}$ & $\begin{array}{c}\text { Fração } \\
\text { Volumétrica } \mathrm{NbC}\end{array}$ & $\begin{array}{c}\text { Tamanho médio } \\
\mathbf{N b C} \text { compactos } \\
\left(10^{-2} \mathrm{~mm}^{2}\right)\end{array}$ & $\begin{array}{c}\text { Fração NbC } \\
\text { compactos }\end{array}$ \\
\hline FFACr & $41 \pm 4$ & - & - & - \\
\hline FFACrNb & $27 \pm 3$ & $2,5 \pm 0,5$ & $7,0 \pm 0,1$ & $64 \pm 2$ \\
\hline
\end{tabular}

A supressão dos carbonetos primários $\mathrm{M}_{7} \mathrm{C}_{3}$, o refinamento da microestrutura e a precipitação dos carbonetos de nióbio $\mathrm{NbC}$, de maior resistência que os primeiros, são considerados fatores que melhoram a resistência ao desgaste abrasivo dos ferros fundidos branco alto cromo $(1,3,7-9,13)$, portanto benéficos a liga produzida para uso em aplicações onde ocorre desgaste abrasivo, como em mineração.

\section{CONCLUSÃO}

A adição de $2 \%$ de nióbio em um FFACr com $25 \% \mathrm{Cr}$ e $3 \% \mathrm{C}$ proporcionou a mudança de uma microestrutura hipereutética para próxima da eutética. Essa adição também promoveu o refinamento dos carbonetos $\mathrm{M7C3}$, principalmente dos carbonetos primários, e da microestrutura do FFACrNb, pela precipitação de $\mathrm{NbC}$. A precipitação dos carbonetos de nióbio, com a adição de $2 \%$, se deu principalmente em sua forma compacta.

\section{Agradecimentos}

Os autores agradecem ao apoio da Fapeming e do CNPq e a Capes pela bolsa. Agradecemos ao Departamento de Engenharia Metalúrgica da UFMG, ao Departamento de Engenharia Mecânica da UFMG e ao PROEX - Pró-Reitoria de Extensão da UFMG pelo suporte dado na realização do trabalho.

\section{REFERÊNCIAS}

1 Tabrett CP, Sare IR, Ghomashchi MR. Microstructure-property relationships in high chromium white iron alloys. Int Mater Rev. 1996;41(2):59-82.

2 Wiengmoon A, Chairuangsri T, Brown A, Brydson R, Edmonds D V., Pearce JTH. Microstructural and crystallographical study of carbides in $30 \mathrm{wt} . \% \mathrm{Cr}$ cast irons. Acta Mater. 2005;53(15):4143-4154.

3 Chung RJ, Tang X, Li DY, Hinckley B, Dolman K. Microstructure refinement of hypereutectic high $\mathrm{Cr}$ cast irons using hard carbide-forming elements for improved wear resistance. Wear. 2013;301(1-2):695-706.

4 Sare IR. Abrasion resistance and fracture toughness of white cast irons. 1979;412-419. 
5 Zum Gahr KH, Scholz WG. Fracture toughness of white cast irons. Jornal of Metals.1980; 32(10):38-44.

6 Fulcher JK, Kosel TH, Fiore NF. The effect of carbide volume fraction on the low stress abrasion resistance of high Cr-Mo white cast irons. Wear. 1983 Feb;84(3):313-325.

7 He-Xing C, Zhe-Chuan C, Jin-Cai L, Huai-Tao L. Effect of niobium on wear resistance of 15\%Cr white cast iron. Wear. 1993;166(2):197-201.

8 Zhi X, Xing J, Fu H, Xiao B. Effect of niobium on the as-cast microstructure of hypereutectic high chromium cast iron. Mater Lett. 2008;62(6-7):857-860.

9 Filipovic M, Kamberovic Z, Korac M, Gavrilovski M. Microstructure and mechanical properties of Fe-Cr-C-Nb white cast irons. Mater Des. 2013;47:41-48.

10 Radulovic M, Fiset M, Peev K, Tomovic M. The influence of vanadium on fracture toughness and abrasion resistance in high chromium white cast irons. J Mater Sci. 1994;29(19):5085-5094.

11 Schindelin J, Rueden CT, Hiner MC, Eliceiri KW. The ImageJ ecosystem: an open platform for biomedical image analysis. Mol Reprod Dev. 2015;82(7-8):518-529.

12 Maratray F, Poulation A. Austenite Retention in High Chromium White Irons. Trans American Foundrymen's Society. 1982;90:795-804.

13 Filipovic M, Kamberovic Z, Korac M, Jordovic B. Effect of Niobium and Vanadium Additions on the As-Cast Microstructure and Properties of Hypoeutectic $\mathrm{Fe}-\mathrm{Cr}-\mathrm{C}$ Alloy. ISIJ Int. 2013;53(12):2160-2166.

14 Coelho GC, Golczewski JA, Fischmeister HF. Thermodynamic calculations for Nbcontaining high-speed steels and white-cast-iron alloys. Metall Mater Trans A. 2003;34(9):1749-1758. 